\title{
Alterations of Contralateral Thalamic Perfusion in Neuropathic Pain
}

\author{
Takahiro Ushida* $^{* 1,3}$, Mitsutaka Fukumoto ${ }^{2}$, Carlos Binti ${ }^{3}$, Tatsunori Ikemoto ${ }^{1}$, \\ Shinichirou Taniguchi, Masahiko Ikeuchi ${ }^{1}$, Makoto Nishihara ${ }^{3}$ and Toshikazu Tani ${ }^{1}$ \\ ${ }^{1}$ Department of Orthopaedic Surgery, and ${ }^{2}$ Department of Radiology, Kochi Medical School, Kochi, Japan; \\ ${ }^{3}$ Multidisciprinary Pain Center, Aichi Medical University, Aichi, Japan
}

\begin{abstract}
Contralateral thalamus, the place of termination of spinothalamic tract, is affected in patients with pain. We employed single photon emission computed tomography (SPECT) to evaluate the thalamic perfusion in patients with spontaneous neuropathic pain. Ten patients with complex regional pain syndrome (CRPS) and eleven radiculopathiy patients were enrolled in this study. Regional cerebral blood flow of thalamus was assessed bilaterally by iodine-123labelled iodoamphetamine SPECT. To standardize the inter-patient data, we set a contralateral thalamic uptake index (CTUI) for assessing thalamic asymmetry. In one study, we found elevation of CTUI in patients with symptoms of neuropathic pain for less than 12 month, whereas no change was observed in the case of a longer lasting disease. An another study demonstrated decrease of CTUI after pain treatment, even though it was unrelated to the pain intensity prior to treatment. Our SPECT study revealed that neuropathic pain altered thalamic neuronal activity. CTUIs were increased in early stage of the disease but decreased as the disease progressed to the chronic stage. These results suggest that CTUI can be used to improve management of neuropathic pain for proper evaluation of spontaneous pain.
\end{abstract}

Keywords: Brain imaging, regional cerebral blood flow, reflex sympathetic dystrophy, pain.

\section{INTRODUCTION}

Although neuromuscular disorders are manifested by a variety of clinical symptoms, pain is among those that are particularly hard to endure. Symptoms of pain are believed to have both central and peripheral origin and were studied with the help of neurophysiological and histocytochemical techniques $[1,2]$. A number of animal models of spinerelated diseases, such as radiculopathy, spinal stenosis, etc., was also introduced to explore the pathways of pain and to examine other related changes [3-5]. These studies were primarily focused on spinal cord due to its accessibility. Limited attention has been paid so far to the brain as well as few studies were undertaken in clinical settings.

In the past decade, several brain imaging techniques, namely single photon emission computed tomography (SPECT), positron emission tomography (PET) and functional MRI (fMRI), emerged as powerful tools used to explore the biology of brain and to diagnose its pathological conditions [6-8]. Since fMRI technology is based on measuring hemodynamic response related to neural activity in the brain, it has advantages in detecting neuroanatomies responded to consecutive functional tasks such as pain stimuli. On the other hand, temporal resolution of SPECT and PET are similarly lower than fMRI technology and beneficial usage of these technologies are rather static brain

\footnotetext{
*Address correspondence to this author at the Multidisciplinary Pain Center, Aichi Medical University, 21 Karimata, Yazako, Nagakute, Aichi , Japan; Tel.: + 81 0561-62-5004; Fax: + 81 0561-62-5004;

E-mail: ushidat-koc@umin.ac.jp
}

activation corresponding to spontaneous pain. Although PET enables better resolution, SPECT, is a more affordable and widely used tool. These techniques are informative, noninvasive and extrapolate brain function from changes in the regional cerebral blood flow (rCBF), since it is spatially and temporally coupled to brain activity. Then, three dimensional data are mapped onto the cerebral anatomy.

It was shown that several brain structures, such as bilateral thalamus, insular cortex, cingulate cortex, primary (SI) and secondary (SII) sensory cortex, are activated by noxious cutaneous stimuli in normal subjects [9-13]. Among them, thalamus is viewed as a particularly important one, because spinothalamic tract, a major pathway of pain, terminates into the medial and lateral thalamic nuclei [14]. Clinical studies, however, reported opposite findings. Iadarola et al. found significant decrease in thalamic activity contralateral to symptomatic side in PET scans of patients with neuropathic pain [15]. Similar results were obtained for the patients with chronic cancer pain [16]. Therefore, one might assume that changes of contralateral thalamic activity and chronic neuropathic pain are presumably linked. In the present study, we used SPECT to examine whether a relationship exists between contralateral thalamic activity and neuropathic pain in patients with CRPS and radiculopathy.

\section{MATERIALS AND METHODOLOGYS}

\section{Subjects}

Twenty-one patients with neuropathic pain including ten with complex regional pain syndrome (CRPS) (seven men and three women; aged 27-65 years; time since the onset of 
symptoms 6-34 month) and eleven with either cervical or lumbar radiculopathy (six men and five women; aged 35-74 years; time since the onset of symptoms 0.3-30 month) who agreed with the study protocol were examined. Twenty two healthy volunteers with matching sex and age served as control. All patients had spontaneous pain and sensory impairments only in unilateral upper or lower extremities. Pain status of each patient was evaluated by the visual analogue scale (VAS). rCBF of the contralateral thalamus was assessed by means of Iodine-123-labelled iodoamphetamine single photon emission computed tomography (SPECT). The absence of previous cerebral vascular and psychological diseases was confirmed using brain computed tomography or magnetic resonance imaging by a psychiatrist not involved into the present study.

All protocols were conducted in accordance with the recommendations outlined in the Declarations of Helsinki and were approved by the local Medical Ethical Committee. All subjects signed an informed consent form prior to the examination.

\section{Procedure}

SPECT scanning started $10 \mathrm{~min}$ after intravenous injection of Iodine-123-labelled iodoamphetamine (111MBq) using an ultra high resolution fanbeam collimators equipped with a triple-detector SPECT device (Toshiba GCA9300A/HG, Tokyo, Japan). Size of field of view used in this study was $409.6 \mathrm{~mm}$ x $409.6 \mathrm{~mm}$. Acquired SPECT images (128 x 128 matrices; $6 \mathrm{~mm}$ slice) were transferred to a Windows PC and then reconstructed from projection data by a filtered backprojection technique with Butterworth and Ramp filters according to Talairach brain atlas.

\section{Measurement of CTUI and Image Analysis}

Activity of contralateral thalamus was evaluated by calculating the contralateral thalamic uptake index (CTUI). Its measurement consisted of the following steps: a) after setting the identical region of interest (ROI) over the both thalami, rCBF corresponding to it was measured bilaterally; b) thalamic perfusion was standardized by subtracting $\mathrm{rCBF}$ of the whole brain to rCBF in ipsilateral and contralateral thalami, respectively; c) CTUI was calculated as the ratio of contralateral to ipsilateral thalamic uptake (Fig. 1A). ROI was then separated into the medial and lateral subdivisions using the three dimensional stereotaxic ROI template (3DSRT) (Fig. 1B). Indexes of both subdivisions (CTMUI and CTLUI respectively) were analyzed employing "NIH image" software (developed at the Research Service Branch (RSB) of the National Institute of Mental Health (NIMH), part of the National Institutes of Health (NIH)). Besides, relations between contralateral thalamic uptake index versus disease duration and pain intensity were examined. In controls, the indexes were calculated as the ratio of left to right consecutive thalamic uptakes.

\section{Statistical Analysis}

Results were analyzed using Wilcoxon matched pairs and Mann-Whitney tests.

\section{RESULTS}

In controls, all CTUI measurements showed symmetric thalamic perfusion $(1.17 \pm 0.63)$.

\section{CTUI and Duration of Disease}

The most significant increase of CTUI was observed in patients with duration of symptoms of pain for less than 12 months. The average of CTUI was $1.94 \pm 1.01, \mathrm{P}=0.0166$ and in some cases even as high as $>3$. In contrast, the average of CTUIs in patients with a longer lasting disease (more than 12 month) was similar to controls (1.06 \pm 0.45$)$ (Fig. 2).

Subdivision analysis was resulted no significant change in both CMTUI and CLTUI in patients with duration of disease for more than 12 month compare to controls. Although not statistically significant $(\mathrm{P}=0.067)$, an increase
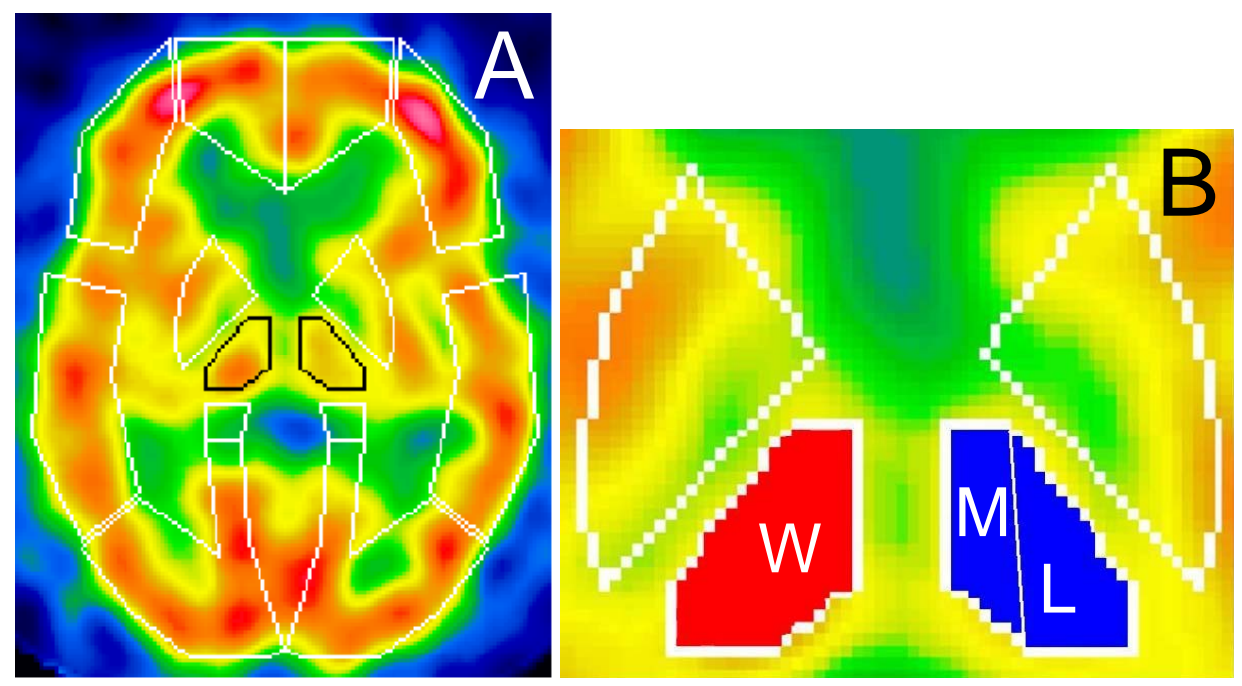

Fig. (1). Standardized brain SPECT images showing presets of ROIs template of 3DSRT (A) and subdivisions of thalamus used for CTUI measurements (B). ROI over thalami is outlined in black. Whole thalamus is marked as (W), medial and lateral parts as (M) and (L) respectively. 


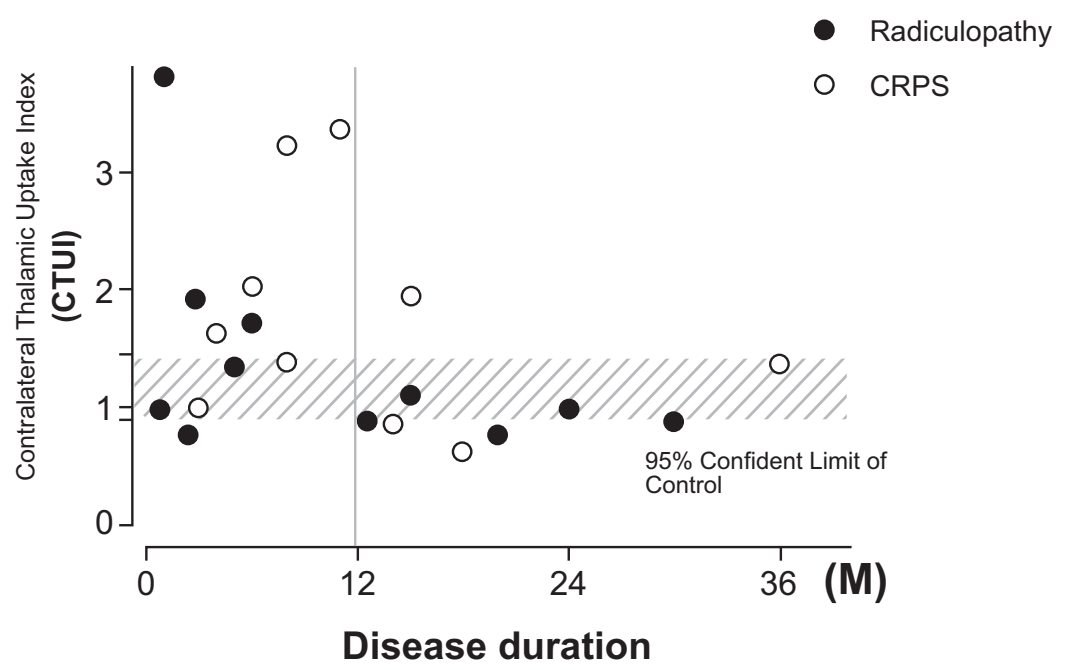

Fig. (2). Scatter gram showing relation between CTUI and disease duration. A significant increase of CTUI is seen for patients with the duration of disease for less than 12 month.

of CMTUI, but not of CLTUI, was observed in patients with duration of disease for less than 12 month (Fig. 3).

\section{CTUI and Intensity of Pain}

In patients with either CRPS or radiculopathy, CTUI and pain intensity, measured by VAS, did not show a clear correlation between each other (VAS=3.5-7.8, average 5,4) (VAS=3.0-7.5, average 4.7), respectively (Fig. 4).

\section{DISCUSSION}

We employed SPECT technique to determine regional concentration of radionuclide in thalamus as a function of time and then to compare its values in normal subjects versus in patients with neuropathic pain. It was observed that contralateral thalamic uptake index (CTUI) is elevated in patients with symptoms of neuropathic pain for less than 12 month. It was also detected that CTUI decreases as a result of pain treatment and that its values in patients with symptoms of pain for more than 12 month are in the range of those in control subjects. This attenuation of the contralateral thalamic activity in chronic pain status has been reported in other investigators. This inhibited thalamic activity might be related to pain pathogenesis, a reversal of this change would be expected as a correlate of pain relief. Accordingly, thalamic hypoactivity has been shown to be reversed by a number of analgesic interventions, from lidocaine blocks to neurosurgical procedures [17-22].

Activation of thalamus in response to acute noxious stimulation as a phenomenon of functional reorganization of central sensory neurons was described previously in both human and animal studies [23-27]. The consensus, however, whether the activation occurs uni- or bi-laterally was not reached. It was also not determined what side of the brain, if uni-laterally, is activated. Our results show that the methodology used to determine thalamic activation by measuring the regional blood flow is of critical importance here. We observed that raw data of thalamic blood flow

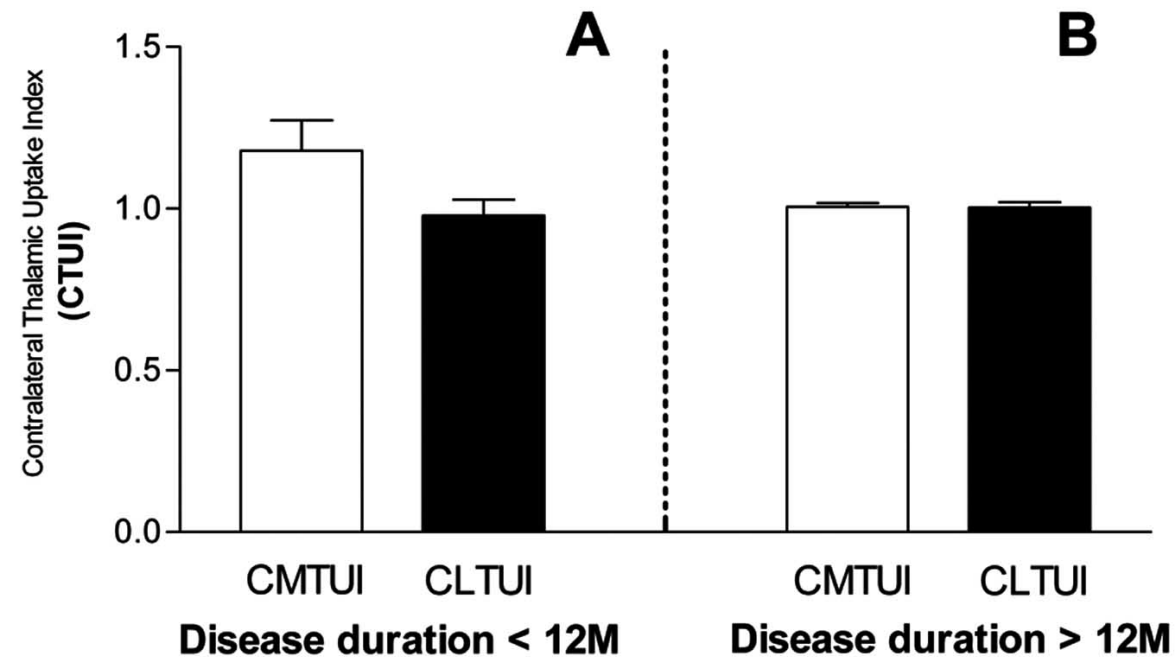

Fig. (3). Changes of CTUI in medial (CMTUI) and lateral (CLTUI) portions of contralateral thalamus in relation to disease duration. An increase of CTUI in medial contralateral thalamus is observed in the case of disease duration for less than 12 month (A). No changes between medial and lateral portions are detected in the case of disease duration for longer than 12 month (B). 


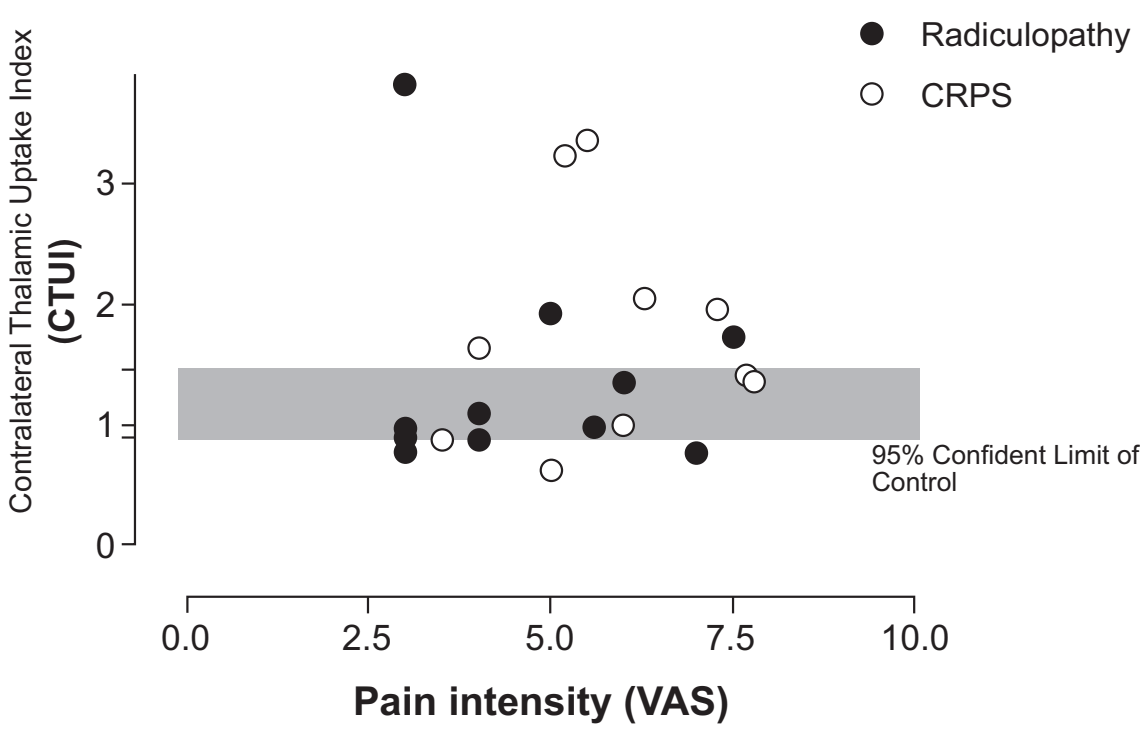

Fig. (4). Scatter gram demonstrating relation between CTUI and pain intensity (VAS). No correlation between pain CTUI and VAS can be found.

obtained from different subjects are not comparable due to significant individual variations. Therefore, we took a different approach and evaluated CTUI by comparing ratios of the total cerebral blood flow against the thalamic blood flow. Using this technique, it was possible to obtain data indicative of an involvement of contralateral thalamus in neuropathic pain. This conclusion is supported by the results of electrophysiological and morphological experiments in primates showing that sensory signals, including noxious inputs, terminate mainly in contralateral thalamus, with less than 10 percent of sensory afferents projecting ipsilaterally [28]. Furthermore, we found an increase of CTUI in the medial portion of contralateral thalamus (CMTUI), but not in the lateral portion, (CLTUI). In this respect, it should be mentioned that medial thalamus is viewed as a portion of thalamus linked to the "affective/motivational" aspect of pain, while lateral is related to "discriminative" pain. Therefore, it is likely that the former aspect of pain sensation is involved the most in patients with neuropathic pain. Additional studies that employ the fine spatial resolution brain imaging tools should help in clarifying this issue further.

Interestingly, we observed that activation of contralateral thalamus depends on the duration of disease and tends to decrease after 12 month since patients report their first complaints. How this observation can be explained? It is possible that sensory cortex adapts to the input of pain in such a way that hyper activation of thalamus for nociceptive transmission and cognition is no longer necessary [29] and /or that continuous pain affects intra-cranial blood distribution and thus results in the sensory blood uncoupling near the activated region [15]. It should be mentioned, however, that the pattern of thalamic reaction in this group of patients is likely to be a very complex issue that requires additional studies considering the possible involvement of other regions of the brain. In recent study, Honda et al. [30] focused on to prefrontal area and cingulate area, and found reduction of cerebral blood flow in chronic pain patients as well.

\section{CONCLUSION}

We utilized contralateral thalamic uptake index (CTUI) to detect changes of thalamic activity in neuropathic pain. CTUIs were increased in the early stage of the disease but decreased as the disease progressed to the chronic stage. Present results suggest that the activity of contralateral thalamus may have a role in development/maintenance of the chronic pain conditions.

\section{ACKNOWLEDGEMENT}

We thank Mr. Naoki Akagi and Mr. Kazuo Morio for technical assistances.

\section{REFERENCES}

[1] Tanaka N, Fujimoto Y, An HS, Ikuta Y, Yasuda M. The anatomic relation among the nerve roots, intervertebral foramina, and intervertebral discs of the cervical spine. Spine 2000; 25(3): 28691.

[2] Heiskari M, Tolonen U, Nystrom SH. Comparison of somatosensory evoked responses from root and cord recorded by skin and epidural electrodes using stimulation of the median nerve in cervical radiculopathy and radiculomyelopathy. Acta Neurochir (Wien) 1986; 79(2-4): 114-9.

[3] Bennett GJ, Xie YK. A peripheral mononeuropathy in rat that produces disorders of pain sensation like those seen in man. Pain 1988; 33(1): 87-107.

[4] Palecek J, Dougherty PM, Kim SH, et al. Responses of spinothalamic tract neurons to mechanical and thermal stimuli in an experimental model of peripheral neuropathy in primates. J Neurophysiol 1992; 68(6): 1951-66.

[5] Olmarker K, Holm S, Rosenqvist AL, Rydevik B. Experimental nerve root compression: A model of acute, graded compression of the porcine cauda equina and an analysis of neural and vascular anatomy. Spine 1991; 16(1): 61-9.

[6] Ogawa S, Lee TM, Nayak AS, Glynn P. Oxygenation-sensitive contrast in magnetic resonance image of rodent brain at high magnetic fields. Magn Reson Med 1990; 14(1): 68-78.

[7] Raichle ME, Martin WR, Herscovitch P, Mintun MA, Markham J. Brain blood flow measured with intravenous H2(15)O. II: Implementation and validation. J Nucl Med 1983; 24(9): 790-8.

[8] Apkarian AV, Stea RA, Manglos SH, Szeverenyi NM, King RB, Thomas FD. Persistent pain inhibits contralateral somatosensory cortical activity in humans. Neurosci Lett 1992; 140(2): 141-7. 
[9] Derbyshire SW, Jones AK, Gyulai F, Clark S, Townsend D, Firestone LL. Pain processing during three levels of noxious stimulation produces differential patterns of central activity. Pain 1997; 73(3): 431-45.

[10] Derbyshire SW, Jones AK. Cerebral responses to a continual tonic pain stimulus measured using positron emission tomography. Pain 1998; 76(1-2): 127-35.

[11] Coghill RC, Talbot JD, Evans AC, et al. Distributed processing of pain and vibration by the human brain. J Neurosci 1994; 14(7): 4095-108.

[12] Casey KL, Minoshima S, Morrow TJ, Koeppe RA. Comparison of human cerebral activation pattern during cutaneous warmth, heat pain, and deep cold pain. J Neurophysiol 1996; 76(1): 571-81.

[13] Casey KL, Minoshima S, Berger KL, Koeppe RA, Morrow TJ, Frey KA. Positron emission tomographic analysis of cerebral structures activated specifically by repetitive noxious heat stimuli. J Neurophysiol 1994; 71(2): 802-7.

[14] Kenshalo DR, Jr, Giesler GJ, Jr, Leonard RB, Willis WD. Responses of neurons in primate ventral posterior lateral nucleus to noxious stimuli. J Neurophysiol 1980; 43(6): 1594-614.

[15] Iadarola MJ, Max MB, Berman KF, et al. Unilateral decrease in thalamic activity observed with positron emission tomography in patients with chronic neuropathic pain. Pain 1995; 63(1): 55-64.

[16] Di Piero V, Jones AK, Iannotti F, et al. Chronic pain: a PET study of the central effects of percutaneous high cervical cordotomy. Pain 1991; 46(1): 9-12.

[17] Katayama Y, Tsubokawa T, Hirayama T, Kido G, Tsukiyama T, Iio M. Response of regional cerebral blood flow and oxygen metabolism to thalamic stimulation in humans as revealed by positron emission tomography. J Cereb Blood Flow Metab 1986; 6(6): 637-41.

[18] Hsieh JC, Belfrage M, Stone-Elander S, Hansson P, Ingvar M. Central representation of chronic ongoing neuropathic pain studied by positron emission tomography. Pain 1995; 63(2): 225-36.

[19] Peyron R, Garcia-Larrea L, Deiber MP, et al. Electrical stimulation of precentral cortical area in the treatment of central pain: electrophysiological and PET study. Pain 1995; 62(3): 275-86.
[20] Pagni CA, Canavero S. Functional thalamic depression in a case of reversible central pain due to a spinal intramedullary cyst: Case report. J Neurosurg 1995; 83(1): 163-5.

[21] Duncan GH, Kupers RC, Marchand S, Villemure JG, Gybels JM, Bushnell MC. Stimulation of human thalamus for pain relief: possible modulatory circuits revealed by positron emission tomography. J Neurophysiol 1998; 80(6): 3326-30.

[22] Garcia-Larrea L, Peyron R, Mertens P, et al. Electrical stimulation of motor cortex for pain control: a combined PET-scan and electrophysiological study. Pain 1999; 83(2): 259-73.

[23] Guilbaud G, Benoist JM, Jazat F, Gautron M. Neuronal responsiveness in the ventrobasal thalamic complex of rats with an experimental peripheral mononeuropathy. J Neurophysiol 1990; 64(5): $1537-54$.

[24] Rampin O, Morain P. Cortical involvement in dorsal horn cell hyperactivity and abnormal behavior in rats with dorsal root section. Somatosens Res 1987; 4(3): 237-51.

[25] Jones EG, Pons TP. Thalamic and brainstem contributions to largescale plasticity of primate somatosensory cortex. Science 1998; 282(5391): 1121-5.

[26] Pons TP, Garraghty PE, Ommaya AK, Kaas JH, Taub E, Mishkin M. Massive cortical reorganization after sensory deafferentation in adult macaques. Science 1991; 252(5014): 1857-60.

[27] Florence SL, Jain N, Pospichal MW, Beck PD, Sly DL, Kaas JH. Central reorganization of sensory pathways following peripheral nerve regeneration in fetal monkeys. Nature 1996 ; 381(6577): 6971.

[28] Willis WD, Kenshalo DR, Jr, Leonard RB. The cells of origin of the primate spinothalamic tract. J Comp Neurol 1979; 188(4): 543 73.

[29] Van Horn JD, Gold JM, Esposito G, et al. Changing patterns of brain activation during maze learning. Brain Res 1998; 793(1-2): 29-38.

[30] Honda T, Maruta T, Takahashi K. Brain perfusion abnormality in patients with chronic pain. Keio J Med 2007; 56(2): 48-52.

(c) Ushida et al.; Licensee Bentham Open.

This is an open access article licensed under the terms of the Creative Commons Attribution Non-Commercial License (http://creativecommons.org/licenses/by-nc/3.0/) which permits unrestricted, non-commercial use, distribution and reproduction in any medium, provided the work is properly cited. 